\title{
Simulación termofluidodinámica en un molde de colada continua de acero
}

\section{Thermofluidodynamic simulation in a steel continuous casting mold}

\author{
Yordy González-Rondón ${ }^{1}$, José Eduardo Rengel ${ }^{2}$, Johnny J. Martínez ${ }^{3}$
}

Recibido: Enero 2021

Aceptado: Agosto 2021

Resumen.- En el presente estudio se resolvió, usando el método de los volúmenes finitos, un modelo matemático multifísico en 3D para predecir los fenómenos de flujo de fluidos y transferencia de calor en un molde de colada continua de palanquillas de acero 20CrMnTi. Los resultados mostraron la alta capacidad de enfriamiento del sistema para la formación de una capa sólida progresiva y uniforme que inicia muy cerca del menisco y alcanza a la salida del molde un espesor del $10 \%$ de la sección de la pieza colada. A la salida de la boquilla de entrada sumergida (SEN) el acero experimentó un reflujo con una profundidad de $0,45 \mathrm{~m}$ medida desde el menisco. Parte del reflujo en ascenso llegó al menisco y descendió infiltrándose por las paredes del molde. Se observaron velocidades menores a $0,2 \mathrm{~m} / \mathrm{s}$ en la zona superior del molde y gran penetración del chorro de acero en el centro del molde. Se concluyó, con base en lo descrito en la literatura, lo siguiente: a) El espesor de la costra solidificada, a la salida del molde, es suficiente para evitar roturas en la pieza debido a la presión ferrostática que ejerce el acero líquido, b) Las velocidades en el área del menisco generan baja turbulencia, lo que evita que se produzca atrapamiento de escoria en el acero líquido, c) Las condiciones fluidodinámicas que se presentan en la zona superior del molde, pueden ser contraproducentes para la transferencia de calor en el menisco y la disipación del sobrecalentamiento en el acero.

Palabras clave: Molde de Colada Continua, Acero, Solidificación, Termofluidodinámica

Summary.-In the present study, a 3D multiphysics mathematical model was solved using the finite volumes method to predict the phenomena of fluid flow and heat transfer in a continuous casting mold of 20CrMnTi steel billets. The results showed the high cooling capacity of the system for the formation of a progressive and uniform solid layer that begins very close to the meniscus and reaches a thickness of $10 \%$ of the section of the casting at the exit of the mold. At the exit of the $S E N$, the liquid steel underwent a reflux with a depth of $0.45 \mathrm{~m}$ measured from the meniscus. Some of the rising reflux reached the meniscus and descended, infiltrating the walls of the mold. Velocities less than $0.2 \mathrm{~m} / \mathrm{s}$, in the upper zone of the mold, and great penetration of the steel jet, in the center of the mold, were observed. It was concluded, based on what is described in the literature, the following: a) the thickness of the solidified crust, at the exit of the mold, is sufficient to avoid breakage in the piece due to the ferrostatic pressure exerted by the liquid steel, c) The fluid dynamic conditions that occur in the upper zone of the mold can be counterproductive for the transfer of heat in the meniscus and the dissipation of overheating in the steel.

Keywords: Continuous Casting Mold, Steel, Solidification, Thermofluidodynamic

\footnotetext{
${ }^{1}$ Ingeniería Mecánica, Universidad de Oriente (Venezuela), yordygonzalez@hotmail.com, ORCID iD: https://orcid.org/0000-0002-4769-7568

${ }^{2}$ Ingeniería Mecánica, Universidad de Oriente (Venezuela), Rengel66@gmail.com, ORCID iD: https://orcid.org/0000-0002-4510-8846

${ }^{3}$ Ingeniería Mecánica, Universidad de Oriente (Venezuela), johnnymartinr@gmail.com, ORCID iD: https://orcid.org/0000-0001-8901-9303
} 
1. Introducción. - La colada continua es el método más importante de producción de acero a nivel mundial, y consiste en hacer que el metal fluya a través de una boquilla de entrada sumergida (SEN) hacia un molde sin fondo refrigerado por agua [1 y 2]. Debido al gradiente de temperatura el acero líquido comienza a solidificar, experimentando la formación de una costra que tiende a adherirse al molde, pero esto se evita con movimientos oscilatorios y con el agregado de polvos de fundición, que se introducen por la parte superior del molde. La costra de acero solidificada alcanza a la salida del molde un espesor de 12 a $25 \mathrm{~mm}[3$ y 4]. En este sentido, el molde tiene como objetivo principal extraer calor del acero líquido en un tiempo muy corto [5 y 6], para lograr que la costra formada resista la presión ferrostática que ejerce el acero líquido sobre ella. Todo este proceso ocurre en un ambiente de complejidades, en primer lugar porque el acero experimenta al mismo tiempo transmisión de calor, flujo de fluidos y transformación de fase [7 y 8]; y en segundo lugar porque se crea una interdependencia entre las variables que participan en el sistema, por ejemplo, si aumenta la velocidad de colada se afecta la infiltración de escoria y ésta a la vez condiciona la lubricación y la transferencia de calor entre la costra sólida y el molde, además aumenta la turbulencia en el flujo de acero líquido causando una distorsión en la región donde el flujo de acero impacta con la costra solidificada [9].

La dinámica de lo que ocurre en el interior de un molde es característico para cada configuración de la SEN - molde y condiciones de operación en el momento de la colada. Esto significa que no es posible crear un modelo general del comportamiento termofluidodinámico del sistema. Además, para un sistema específico en donde las condiciones de operación varían con el tiempo, tampoco es posible hacer generalidades. Debido a estas dificultades recientemente se han hecho esfuerzos por comprender lo que ocurre en el interior de un molde de colada continua. Tkadlečková et al. [10], realizaron un modelado numérico de la solidificación de productos redondos mediante la colada continua de acero inoxidables, para observar el campo de temperatura, la longitud metalúrgica y el grosor de la capa al final del molde. Jiang et al. [11], simularon la solidificación del acero y observaron que una parte del acero líquido que salía de la buza se regresaba hacia el menisco e impactaba directamente en la interfaz de la escoria de acero, y la otra parte se movía hacia abajo del frente de solidificación formando un par de vórtices, que a medida que se extraía el calor latente del molde, la costra solidificada crecía gradualmente y la temperatura del líquido disminuía con el flujo de fluidos. Long et al. [12], desarrollaron un modelo híbrido 3D/2D para investigar los fenómenos de flujo de fluidos y solidificación durante la colada continua y concluyeron que el efecto de impacto causado por el chorro de acero fundido en la formación de la costra de solidificación era significativo. Zhang et al. [13], desarrollaron un modelo acoplado de flujo de fluidos, transferencia de calor y solidificación mediante el software ANSYS Fluent, en los resultados observaron que el acero líquido fluía en el molde creando recirculaciones tanto en la parte inferior como en la superior muy cerca del menisco, además determinaron que la escoria líquida aprovecha la oscilación del molde para infiltrarse por un espacio entre el molde y la costra solidificada. Saldaña et al. [14], analizaron la profundidad de inmersión de la boquilla de entrada sumergida en las oscilaciones del menisco y determinaron que a medida que aumentaba la profundidad de inmersión de la SEN, había una disminución en la intensidad y la velocidad del flujo dirigida hacia la superficie libre y que era posible encontrar un intervalo de funcionamiento adecuado para la profundidad de inmersión de la buza. Ni et al. [15], en su análisis sobre la profundidad de la SEN dedujeron que un aumento en la profundidad de inmersión disminuye la velocidad interfacial y esto reduce el riesgo de arrastre de escoria, además la temperatura próxima al área del menisco disminuye ligeramente. Zhang et al. [16], desarrollaron un modelo matemático multifísico 3D mediante el software comercial ANSYS Fluent y mostraron el comportamiento de las líneas de flujo, la formación de la costra de acero y los perfiles de velocidad para el sistema molde - SEN con y sin acoplamiento magnético. Trindade et al. [17], determinaron mediante simulaciones numéricas que la costra que se generaba en la parte superior del molde cerca del centro del agitador magnético era muy delgada y que en el centro de la palanquilla donde no influía 
el agitador la costra era más gruesa. Sun et al. [18], publicaron que al combinar un sistema de agitación electromagnética (EMS) con frenado electromagnético (EMBR) era posible cambiar el patrón de flujo, la uniformidad, la actividad del acero fundido en la región superior del molde y la profundidad de penetración de las inclusiones en un molde. Yan et al. [19], mediante simulaciones numérica revelaron que luego que la escoria líquida se infiltraba por el menisco durante el ascenso del molde, una gran cantidad de esta escoria se regresaba al reservorio de escoria cuando el molde descendía (en la mitad del período de la tira negativa), además que el crecimiento de la costra era más predominantemente en el período que el molde descendía y menos predominante cuando el molde ascendía.

Si bien se ha avanzado en el desarrollo de modelos matemáticos, experimentación en prototipos a escala y prácticas en plantas industriales, aún falta mucho por investigar, para comprender con precisión todos los fenómenos que ocurren en las distintas configuraciones del proceso de colada continua de acero, un ejemplo de éstos, es el efecto que tiene, en el molde de palanquillas, el flujo de fluidos y la transferencia de calor sobre la formación de ciertos defectos que van en detrimento de la calidad del acero. Por tal razón, en este estudio se desarrolla un modelo matemático multifísico 3D de un molde de colada continua de acero mediante el software ANSYS Fluent, para simular el comportamiento transitorio de transferencia de calor y flujo de fluidos en un molde de palanquillas y buza sumergida circular con un solo orificio. El modelo computacional desarrollado se valida con el método analítico y el resultado del comportamiento de la costra solidificada de acero formada en el molde, se compara con aquel publicado por Zhang et al. [16]. En el análisis de los resultados, se presta especial atención en comprender los perfiles de temperatura y velocidad del acero en el molde, así como las líneas de flujo y vectores de velocidad. Los resultados aquí reportados, serán de gran utilidad para que, en trabajos posteriores, se avance en condiciones termofluidodinámicas que conlleven a un producto de altísima calidad.

\section{Materiales y métodos. -}

2.1 Suposiciones básicas. - Las suposiciones se deben especialmente a la complejidad del sistema, dado que ocurren simultáneamente varios fenómenos que hacen que los recursos computacionales sean limitados. De manera que las siguientes condiciones se asumieron para simplificar el modelo numérico y hacerlo computacionalmente más eficiente:

- Se trató el acero fundido como un fluido newtoniano incompresible con parámetros termofísicos constantes, como la densidad, la viscosidad, la conductividad térmica y el calor específico.

- No se consideró la influencia de la conicidad y la oscilación del molde.

- La superficie libre del acero en el molde fue plana y adiabática.

- Las paredes del molde se consideraron estacionarias y a la costra solidificada se le especifico una velocidad igual a la velocidad de colada.

- Las condiciones de velocidad a la entrada y salida del molde se aplicaron para mantener la velocidad de flujo en el molde.

- El sistema SEN - molde representó un proceso convencional de colada continua vertical de molde recto.

2.2 Modelo de flujo de fluidos. - Las ecuaciones de continuidad y de Navier-Stokes de conservación del momento transitorio se pueden expresar de la siguiente manera:

$$
\begin{gathered}
\nabla \cdot \vec{v}=0 \\
\frac{\partial}{\partial t}(\rho \vec{v})+\nabla \cdot(\rho \vec{v} \vec{v})=-\nabla p+\nabla \cdot\left[\mu\left(\nabla \vec{v}+\nabla \vec{v}^{T}\right)\right]+\rho \vec{g}+S
\end{gathered}
$$


donde, $\rho$ es la densidad, $\vec{v}$ es la velocidad del fluido, $p$ es la presión estática, $\mu$ es la viscosidad efectiva y $\mu$ es la suma de la viscosidad dinámica $\left(\mu_{1}\right)$ y la viscosidad turbulenta $\left(\mu_{\mathrm{t}}\right)$. Para la solidificación, se utilizó la técnica de entalpía-porosidad donde la zona blanda se trató como un medio pseudoporoso y la porosidad en cada celda fue igual a la fracción líquida en esa celda. La porosidad del medio poroso indica el contenido líquido en sólido o líquido dispersado a través de las dendritas sólidas.

Cuando la porosidad de la celda es igual a cero, la celda es tratada como completamente solidificada y la velocidad en esta zona se extingue ya que no hay líquido. Para tener en cuenta la presencia de material sólido y mover la zona solidificada en la dirección de la colada, el término fuente " $S$ " se agregó al lado derecho de la ecuación de Navier-Stokes. Esto mueve el material recién solidificado a una velocidad de tracción constante, la cual se estableció para que fuese igual a la velocidad de colada. El término fuente se expresa como:

$$
S=\frac{(1-\beta)^{2}}{\left(\beta^{3}+\xi\right)} A_{m u s h}\left(\vec{v}-\vec{v}_{p}\right)
$$

donde, $\beta$ es la fracción líquida, $\xi$ es una pequeña constante positiva que se proporciona en el denominador para evitar la división entre cero $(0,001)$ y $A_{\text {mush }}$ denota la zona blanda constante y está influenciada por la morfología del grano en el frente de solidificación. El valor de la constante de la zona blanda se considera entre $10^{4}$ y $10^{8}$. En el término fuente " $S$ ", se usa la velocidad relativa entre el líquido fundido y el sólido $\left(\vec{v}_{p}\right)$ en lugar de la velocidad absoluta del líquido.

Para considerar los efectos de la turbulencia en el flujo de fluidos del molde, autores [20 y 21] han informado que el modelo de turbulencia $k-\varepsilon$ realizable es el más adecuado para el modelado de solidificación. Este modelo de turbulencia $k-\varepsilon$ realizable evita la singularidad para un valor bajo de turbulencia [21 y 22], que se puede encontrar en la zona blanda. Las ecuaciones diferenciales parciales para la energía cinética turbulenta $(k)$ y la tasa de disipación $(\varepsilon)$ están dadas por:

$$
\begin{gathered}
\rho \frac{\partial k}{\partial t}+\nabla \cdot(\rho k \vec{v})=\nabla \cdot\left[\left(\mu_{l}+\alpha_{k} \mu_{t}\right) \nabla k\right]+G+\rho \varepsilon+S_{k} \\
\rho \frac{\partial \varepsilon}{\partial t}+\nabla \cdot(\rho \varepsilon \vec{v})=\nabla \cdot\left[\left(\mu_{l}+\alpha_{\varepsilon} \mu_{t}\right) \nabla \varepsilon\right]+C_{1 \varepsilon} \frac{\varepsilon}{k} G-C_{2 \varepsilon} \rho \frac{\varepsilon^{2}}{k+\sqrt{u \varepsilon}}+S_{\varepsilon}
\end{gathered}
$$

donde, $\alpha_{k}$ y $\alpha_{\varepsilon}$ son los números de Prandtl efectivos inversos, $C_{1 \varepsilon}=1.44$ y $C_{2 \varepsilon}=1.92$ [23 y 24 ] son los parámetros del modelo, $G$ es la generación de la energía cinética de turbulencia debido al gradiente de velocidad media. Los términos fuente $S_{k}$ y $S_{\varepsilon}$ se agregan a las ecuaciones de turbulencia cinética y disipación, en las zonas blanda y solidificada para dar cuenta de la presencia de materia sólida, y se pueden expresar de la siguiente manera:

$$
S_{\varnothing}=\frac{(1-\beta)^{2}}{\left(\beta^{3}+\xi\right)} A_{m u s h} \emptyset
$$

donde, $\emptyset$ es la cantidad de turbulencia, que resuelve $(k, \varepsilon)$.

2.3 Modelo de solidificación. - La entalpía del material se calculó como la suma de la entalpía sensible, $h$, y el calor latente, $\Delta H$ :

$$
H=h+\Delta H
$$

siendo;

$$
h=h_{\text {ref }}+\int_{T_{\text {ref }}}^{T} c_{p} d T
$$

donde, $h_{r e f}$ es la entalpía de referencia, $T_{r e f}$ es la temperatura de referencia, $c_{p}$ es el calor específico a presión constante. 
La fracción líquida $\beta$ se puede definir como:

$$
\left\{\begin{array}{c}
\beta=0 \text { si } T \leq T_{\text {solidus }} \\
\beta=\frac{T-T_{\text {solidus }}}{T_{\text {liquidus }}-T_{\text {solidus }}} \text { si } T_{\text {solidus }}<T_{\text {liquidus }} \\
\beta=1 \text { si } T \geq T_{\text {liquidus }}
\end{array}\right.
$$

El contenido de calor latente ahora se puede escribir en términos del calor latente del material, $L$, de la siguiente manera:

$$
\Delta H=\beta L
$$

El contenido de calor latente puede variar entre cero (para un sólido) y 1 (para un líquido). La ecuación de la conservación de la energía es dada por:

$$
\frac{\partial}{\partial t}(\rho H)+\nabla \cdot(\rho v H)=\nabla \cdot(\alpha \nabla T)+S
$$

donde, $H$ es la entalpía, $\rho$ es la densidad, $v$ es la velocidad del fluido, $\alpha$ es la conductividad térmica y $S$ es el término fuente.

2.4 Modelo geométrico y mallado. - Para calcular numéricamente el comportamiento de la transferencia de calor y y el flujo de fluidos en el molde de la colada continua se construyó un modelo numérico del molde de palanquillas mediante el programa ANSYS Fluent. La figura I muestra el diseño del modelo computacional en $3 \mathrm{D}$, formado por la $\mathrm{SEN}$ y el molde de sección transversal $0,16 \times 0,16 \mathrm{~m}$ y longitud de $0,8 \mathrm{~m}$. La profundidad de la buza sumergida o longitud de inmersion fue de $0,1 \mathrm{~m}$ y los diámetros interno y externo fueron $0,033 \mathrm{~m}$ y $0,09 \mathrm{~m}$, respectivamente. Para simular el comportamiento de la costra solidificada con mayor precisión, se realizó un refinamiento local de la malla con un número de nodos de 654.956 y 642.447 elementos. En la evaluación de la malla, se obtuvo que la calidad ortogonal fue de 0,52618 encontrándose por encima del límite inferior $(0,20)$, la máxima oblicuidad fue 0,68916 valor inferior a 0,8 para calificarse como buena, la relación de aspecto fue de 18,383 y está dentro del intervalo 10 - 100 donde se considera aceptable, y para la calidad de mallado por metodo de elemento el máximo número fue de 0,90427 siendo un valor muy cerca de 1 para considerase como buena. El método de solución del acoplamiento de presión - velocidad, se basó en el algoritmo SIMPLEC [13, 16] y el paso del tiempo fue constante en cada iteración $(0,001)$. El tiempo de simulación fue de 196 horas en un computador Core i7 de ocho núcleos y 16 GB de memoria Ram. Los criterios de convergencia de la simulación se establecieron con los residuos de todas las variables dependientes en 1x10-6 en cada paso de tiempo.

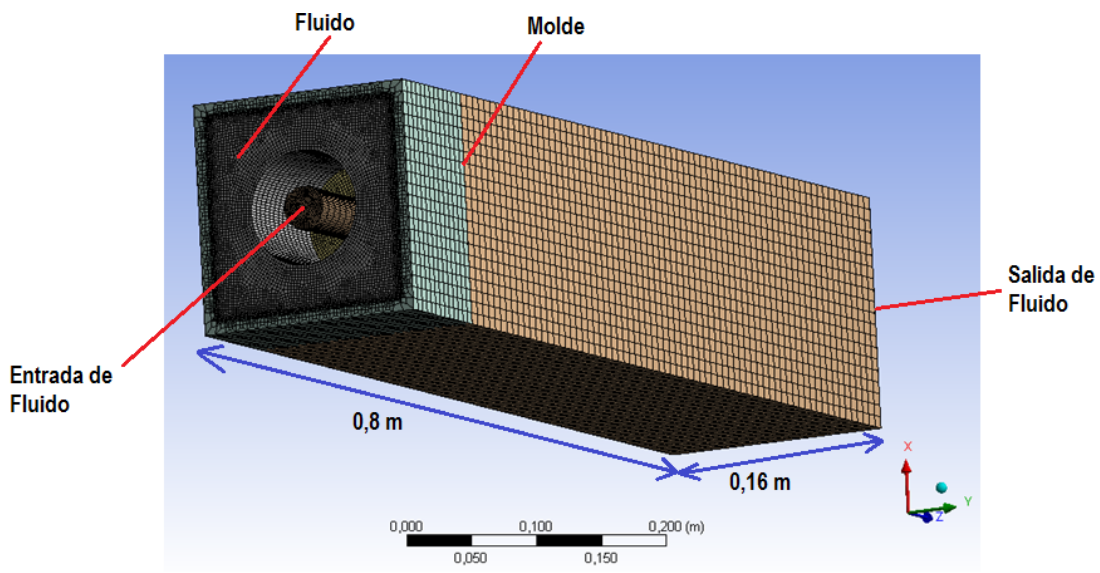

Figura I. Modelo geometrico y mallado de la palanquilla

Memoria Investigaciones en Ingeniería, núm. 21 (2021). pp. 29-42

https://doi.org/10.36561/ING.21.4

ISSN 2301-1092 • ISSN (en línea) 2301-1106 
2.5 Condiciones de simulación. - El método de la colada continua de acero que se presenta en este trabajo es vertical. La entrada fue definida en la parte superior de la buza, allí la velocidad se calculó mediante la conservación de la masa del sistema buza - molde, y la temperatura esta representada por la sumatoria de la temperatura de liquidus más el sobrecalentamiento. La salida se fijó en la parte inferior del molde y la condición de borde en esta zona fue caracterizada como outflow. Se consideró que la costra solidificada se movia a la velocidad de extracción de la palanquilla. En esta investigación se consideraron ciertas condiciones utilizadas por Zhang et al. [16]. El acero es de bajo carbono 20CrMnTi y las composiciones químicas, los parámetros del proceso y las propiedades físicas del acero se describen en detalle en las tabla I y II.

\begin{tabular}{ccccccccc}
\hline Elementos & $C$ & $M n$ & $S i$ & $P$ & $S$ & $N i$ & $C r$ & $C u$ \\
\hline Masa (\%) & 0,19 & 0,89 & 0,24 & 0,016 & 0,001 & 0,01 & 1,08 & 0,01 \\
\hline
\end{tabular}

Tabla I. Composición química del acero 20CrMnTi ${ }^{[16]}$.

\begin{tabular}{|c|c|c|c|}
\hline Parámetros del proceso & Valores & Propiedades fisicas del acero & Valor \\
\hline Sección de la palanquilla $/ \mathrm{m}^{2}$ & $0,160 \times 0,160$ & Temperatura de liquidus / $\mathrm{K}$ & 1.783 \\
\hline Longitud del molde /m & 0,800 & Temperatura de solidus / $\mathrm{K}$ & 1.757 \\
\hline Diámetro interno SEN /m & 0,033 & Densidad $/ \mathrm{kg} \cdot \mathrm{m}^{-3}$ & 7.000 \\
\hline Diámetro externo SEN /m & 0,090 & Viscosidad $/ \mathrm{kg} \cdot \mathrm{m}^{-1} \cdot \mathrm{s}^{-1}$ & 0,0062 \\
\hline Longitud inmersión SEN /m & 0,100 & $\begin{array}{c}\text { Conductividad térmica /W.m } \\
{ }^{1} \cdot \mathrm{K}^{-1}\end{array}$ & 31 \\
\hline Velocidad de colada $/ \mathrm{m} \cdot \mathrm{min}^{-1}$ & 1,700 & Calor específico $/ \mathrm{J} \cdot \mathrm{kg}^{-1} \cdot \mathrm{K}^{-1}$ & 650 \\
\hline Temperatura de colada /K & 1.803 & Calor latente de fusión $/ \mathrm{J} \mathrm{kg}^{-1}$ & 250.000 \\
\hline $\begin{array}{l}\text { Temperatura del agua de } \\
\text { enfriamiento } / \mathrm{K}\end{array}$ & 300 & $\begin{array}{l}\text { Coeficiente de expansión } \\
\text { térmica } / 1 . \mathrm{kg}^{-1}\end{array}$ & 0,002 \\
\hline Temperatura ambiente /K & 300 & - & - \\
\hline
\end{tabular}

Tabla II. Parámetros del proceso y propiedades fisicas del acero ${ }^{[16]}$.

\section{Resultados y discusión.-}

3.1 Validación del modelo. - Para la validación del modelo numérico se consideró un proceso de conducción de calor transitorio bidimensional en el molde, donde la figura II representa la vista superior del molde en el plano XY.

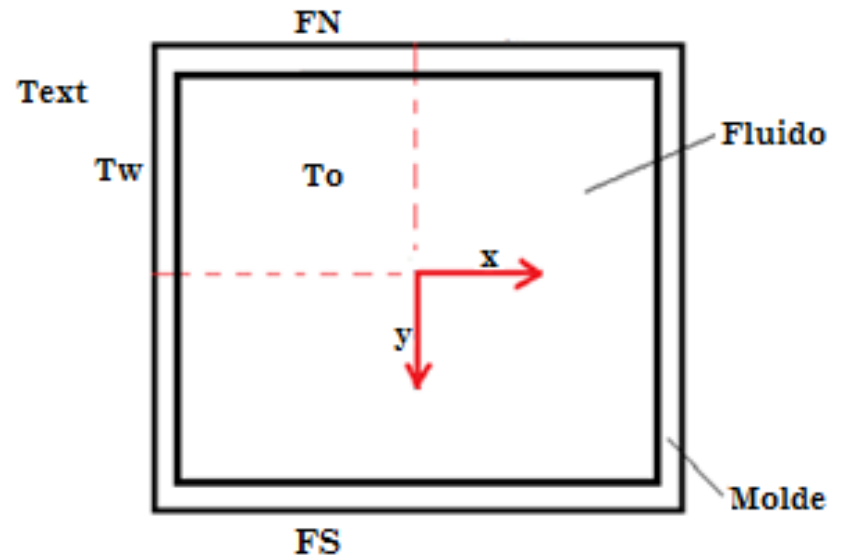

Figura II. Zona bidimensional del sistema molde - acero.

Memoria Investigaciones en Ingeniería, núm. 21 (2021). pp. 29-42

https://doi.org/10.36561/ING.21.4

ISSN 2301-1092・ISSN (en línea) 2301-1106 


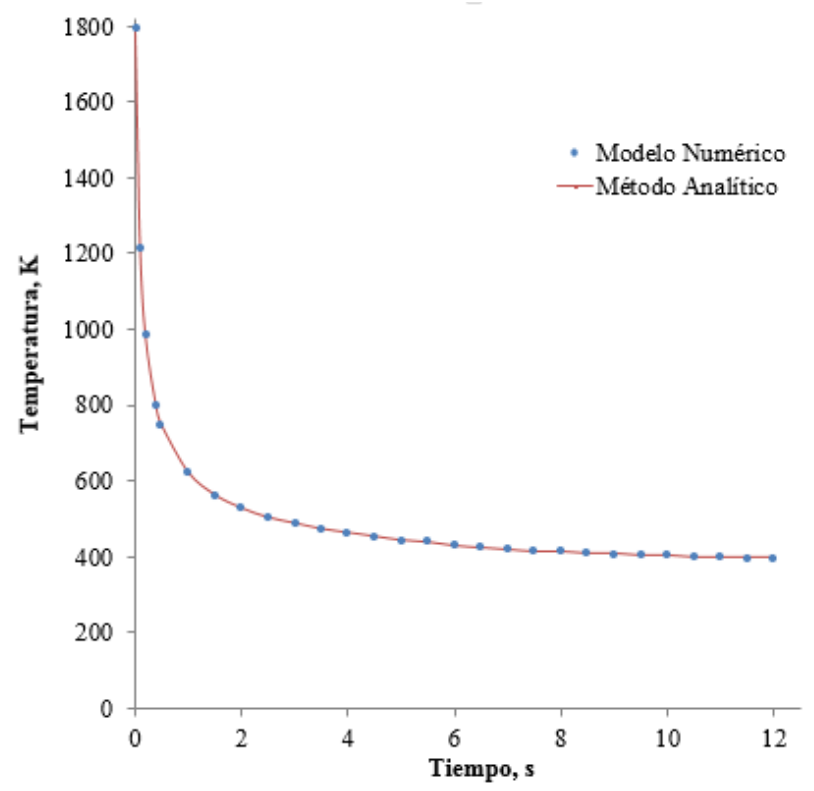

Figura III. Variación temporal de la temperatura a $1 \mathrm{~mm}$ de la pared del molde.

La conducción de calor transitoria para un sólido se define por:

$$
\frac{\partial}{\partial t}(\rho H)=\nabla \cdot(\alpha \nabla T)
$$

La ecuación 12 no puede resolverse analíticamente, sin embargo, considerando la figura II del sistema molde - acero y asumiendo que:

- $\quad$ Las fronteras norte (FN) y sur (FS) están aisladas para convertir el problema 2D en 1D.

- El molde en la dirección x es lo suficientemente largo como para que en el centro no cambie la temperatura durante el proceso (sistema semiinfinito).

- $\quad$ Las propiedades termofísicas no cambian con la temperatura.

La ecuación 12 ahora puede ser resuelta analíticamente, quedando de la siguiente manera:

$$
\frac{T-T_{w}}{T_{o}-T_{w}}=f e r\left(\frac{x}{2 \sqrt{\alpha t}}\right)
$$

donde, $f \operatorname{er}($ ) es la función error de Gauss definida como la integral de una distribución normal y $\alpha$ es la difusividad térmica, $t$ es el tiempo de simulación, $T_{\text {ext }}$ es la temperatura del agua de enfriamiento del molde, $T_{o}$ es la temperatura del acero, $T$ es la temperatura a una distancia $x$ muy cerca del molde y $T_{w}$ es la temperatura en la pared del molde.

Para la validación se determinaron los cambios de temperatura en la dirección $\mathrm{x}$ a una distancia de $1 \mathrm{~mm}$ de la pared del molde en función del tiempo. En la figura III se muestra las variaciones temporales de las temperaturas obtenidas con el modelo numérico desarrollado en esta investigación y la solución analítica de la ecuación 13. Se puede observar que ambas curvas tienen igual comportamiento, por lo que se confirma que la solución numérica es consistente y el modelo queda validado, sin embargo, es de resaltar que la manera de comprobar que el comportamiento de las temperaturas obtenidas esté muy cercanas a la realidad, es hacer comparaciones con resultados experimentales, debido a las simplificaciones asumidas en la metodología de validación. 
3.2 Transferencia de calor. - La Figura IVa muestra la sección transversal de la palanquilla en el plano YZ y distintos cortes de la sección transversal en el plano XY localizadas a 0,001 m, 0,5 m y $0,8 \mathrm{~m}$ desde el menisco. Se observa en el plano YZ el crecimiento del espesor de la costra sólida que experimenta el acero durante su estadía en el molde, debido al difrerencial de temperatura entre el molde refrigerado y el acero colado. En los planos XY se muestran que la fundición comienza a solidificar muy cerca del menisco, aumentando el espesor de manera significativa y uniforme en el sentido de la dirección del proceso de colada. Los contornos de temperatura en los planos XY indican que la solidificación del acero ocurre simétricamente en el molde y que en las aristas de la palanquilla, el espesor de la costra sólida es mayor que en otras zonas. En la figura IVb se describe como en la interfaz que forma el molde y la fundición de acero, en la zona del menisco, hay una disipación del calor acelerada, lo que hace que la costra sólida comience a formarse cerca del menisco. Sin embargo, este fenómeno no es el más idóneo, porque lo más recomendable es que en la zona del menisco el fluido permanezca a una temperatura mayor a la de solidificación para retrasar el inicio de la formación de la costra y asi evitar que la zona superior del molde tienda a solidificarse [25]. Tambien se observa en la figura IVb que en las aristas del molde se encuentran las más bajas temperaturas en comparación con el centro de las caras del molde, y esto se debe a que en las aristas ocurre enfriamiento en ambas caras, lo que hace que el espesor de la costra de acero en las aristas sea mayor a las que se forman en las demas zonas de las caras del molde.

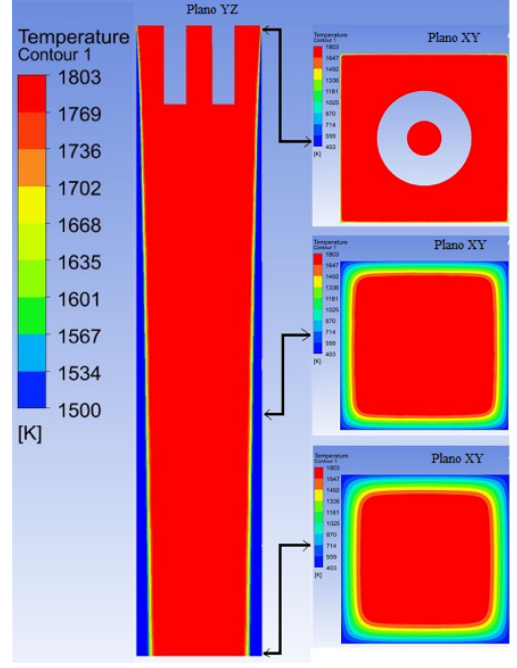

a)

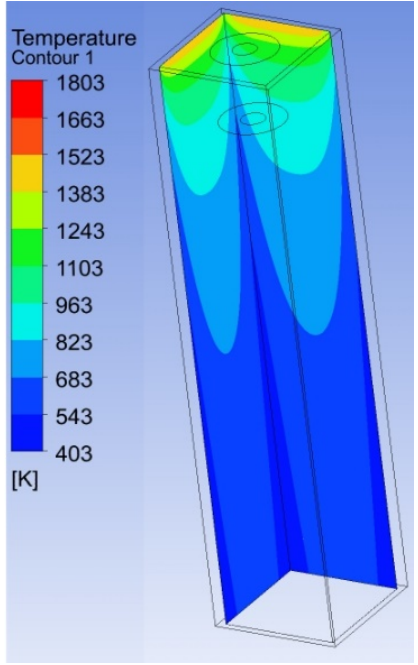

b)

Figura IV. Contornos de tempeartura en la costra sólida: a) Plano YZ y cortes transversales en el Plano XY a las distancias desde el menisco a 0,001 m, 0,5 m y 0,8 m, b) interfaz fluido - molde.

En la figura V se compara el crecimiento de la costra solidificada de acero entre los resultados arrojados por Zhang et al.[16] y los obtenidos en esta investigación con el modelo numérico desarrollado. Se observa gran similitud en ambas investigaciones, con igual tendencia del crecimiento del acero solidificado, coincidiendo que el máximo espesor de la costra a la salida del molde es aproximadamente $0,016 \mathrm{~m}$, lo que representa un valor suficiente para que la costra no se fracture y se evite el desgarro del acero líquido [1]. Es de resaltar, que en este tipo de colada convencional el sistema tiene alta capacidad de enfriamiento, debido a que el acero fundido en el molde solidifica rápidamente y el espesor de la costra sólida aumenta de manera significativa y uniforme. 


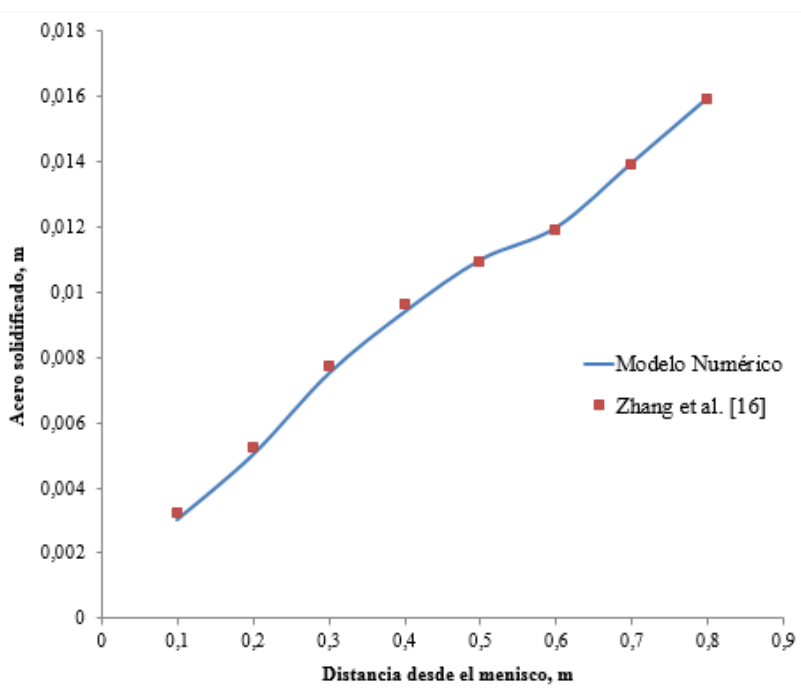

Figura V. Crecimiento de la costra sólida de acero.

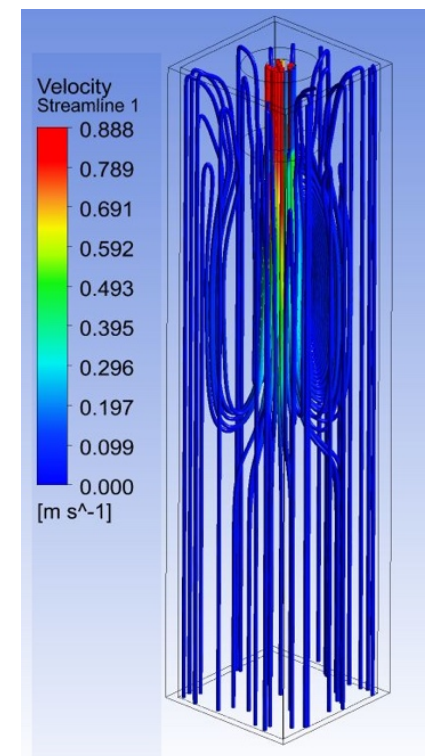

a)

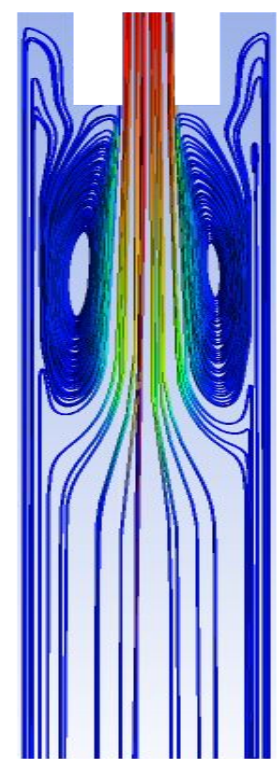

b)

Figura VI. Lineas de flujo en el molde de colada: a) Distribución 3D, y b) $2 D$ en el plano YZ.

3.3 Flujo de fluidos. - La figura VIa describe la trayectoria que experimenta el flujo de acero en el molde. El metal sale de la SEN y se introduce con gran impulso en el molde hasta una profundidad de aproximadamente de $0,45 \mathrm{~m}$ en la dirección de colada. A medida que el acero fluye, el chorro arrastra el fluido circundante debido a la fricción, reduciendo el impulso y aumentando el ancho del chorro. Mientras tanto, la presión disminuye en la región cercana al flujo descendente del chorro. Todo este proceso conduce a un reflujo vertical desde la salida de la SEN hasta la región media del molde (ver Figura VIb), y en la sección transversal, en el plano XY, desde el centro de la palanquilla hasta las paredes del molde. Parte del flujo rotacional llega cerca del menisco para luego descender por la región de las paredes del molde. 


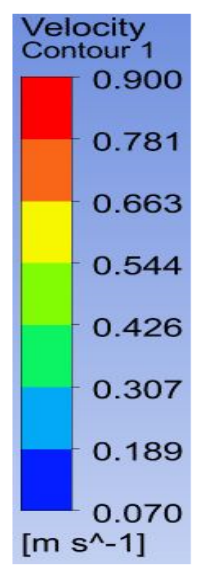

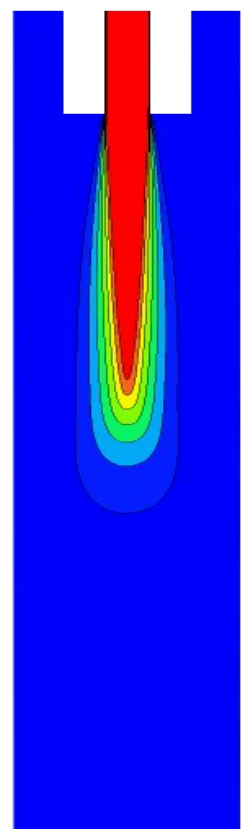

a)
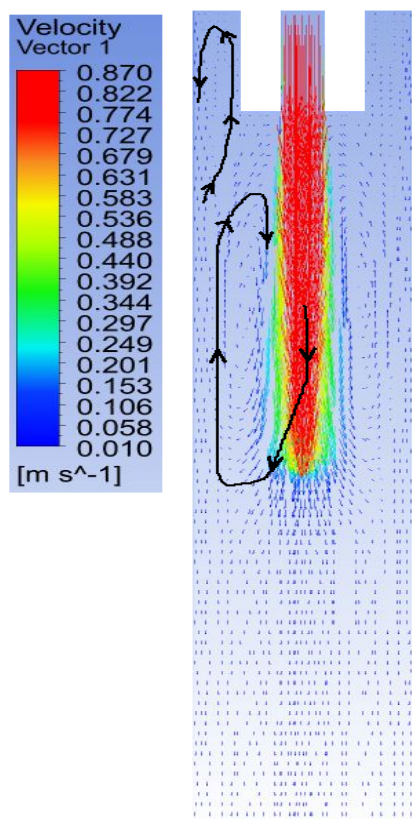

b)

Figura VII. Velocidad del fluido en el plano YZ: a) Contorno de velocidad, y b) Vectores de velocidad.

La Figura VIIa muestra los contornos de velocidad del flujo de acero en un plano vertical en el centro del molde, específicamente en el plano YZ, según los ejes de coordenadas descritos en la figura I. Se observa que el flujo de acero se desplaza en línea recta en el sentido de avance de la palanquilla, obteniéndose los mayores velocidades en la buza sumergida y en el centro de la pieza, hasta una distancia desde el menisco de aproximadamente de 0,45 m. Este comportamiento del fluido se describe muy bien en la figura VIIb, donde la magnitud de los vectores de velocidad indican que el chorro de acero alcanza gran profundidad en el centro de la palanquilla con una rapidez de $0,8 \mathrm{~m} / \mathrm{s}$. Sin embargo, esta elevada velocidad no ayudaría a la eliminación de inclusiones en el molde, porque no habría tiempo a que sean atrapadas en la parte superior del molde. Tambien afectaría la disipación del sobrecalentamiento del acero, ya que lo recomendable es que el sobrecalentamiento sea extraído en el molde y no en la zona de enfriamiento secundario porque alargaría la longitud metalúrgica [25]. Por otro lado, cerca de las paredes del molde, donde se forma la costra sólida, se registraron velocidades de $0,2 \mathrm{~m} / \mathrm{s}$, las cuales son muy bajas para estas regiones, porque se necesitan altas velocidades para romper las dendritas de la interfaz de solidificación y promover el nucleado, y asi conducir a una mejora en la transición de una solidificación columnar a una solidificación equiaxial [15].

Tambien es importante resaltar que en la figura VII se reportaron bajas velocidades en la parte superior del molde, cerca del menisco. Este efecto pudiera ser contraproducente para la transferencia de calor en el menisco, debido a que se produciría una solidificación muy temprana en el menisco, lo que formaría un borde grueso que generaría problemas de calidad como marcas profunda de oscilación, grietas y otros defectos superficiales [25]. Sin embargo, esta situación pudiera ser favorable para el acero porque las bajas fluctuaciones en el área del menisco reducen el atrapamiento de escoria de polvos coladores [9]. 


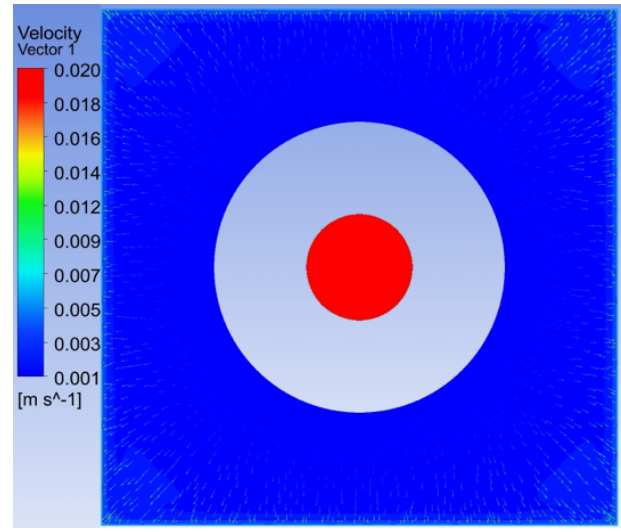

a)

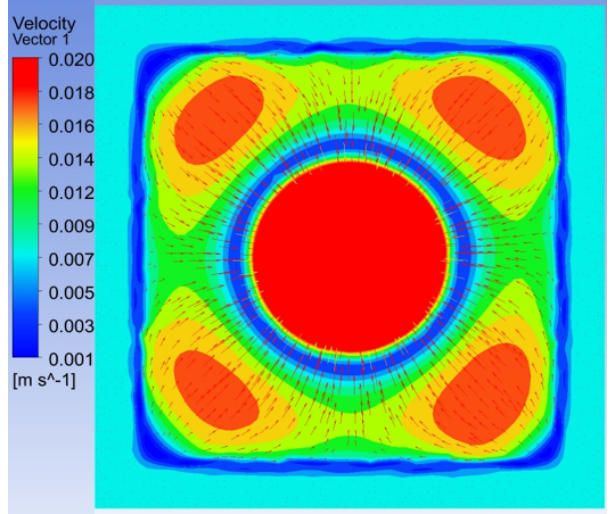

b)

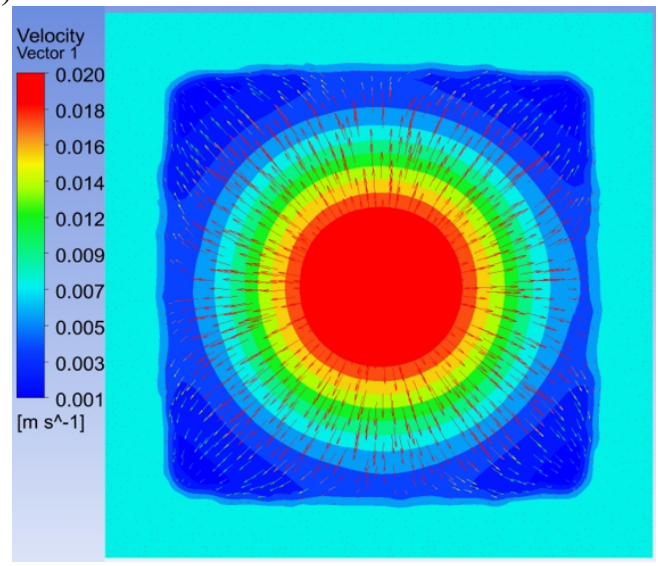

c)

Figura VIII. Contornos y vectores de velocidad tangencial en el plano XY: a) a 0,005 m del menisco, b) a 0,2 m del menisco, y c) a 0,483 m del menisco.

La figura VIII muestra las velocidades tangenciales del flujo de acero en tres secciones transversales en el plano XY, con diferentes profundidades de molde. Se puede ver, que en la figura VIIIc a una profundidad de $0,483 \mathrm{~m}$ desde el menisco los vectores tangenciales alcanzan una mayor magnitud en el centro de la palanquilla y disminuyen a medida que se desplazan hacia las paredes del molde para iniciar el reflujo de forma ascendente. Luego a una profundidad de $0,2 \mathrm{~m}$ desde el menisco (figura VIIIb) los vectores tangenciales se desplazan desde las paredes del molde hacia el centro de la palanquilla para cumplir con la recirculación que experimenta el chorro en el molde como se mostró en la figura VIIb. Parte del fluido que no recircula entre $0,2 \mathrm{~m}$ y $0,483 \mathrm{~m}$ desde el menisco, continúa hacia la parte superior del molde por la zona de la buza y luego se desplaza hacia las paredes del molde, como se muestra en la figura VIIIa, por donde se infiltra para iniciar el descenso. Es importante resaltar, que las tres secciones transversales mostrada en la figura VIII permiten suponer que la distribución de la velocidad tangencial del acero en el molde es simétrica y que los reflujos que se forman en el molde son uniformes. Sin embargo, de haber una modificación de la geometría del puerto de salida debido al desgaste de la SEN ocasionada por erosión y por el efecto clogging, se afectaría el patrón de flujo en el molde, que seguramente producirían distorciones en el flujo de fluidos [14 y 26]. 
4. Conclusiones. - Mediante simulaciones numéricas se investigó el comportamiento de la transferencia de calor y el flujo de acero en un molde de colada continua convencional. Se analizó la formación de la costra sólida, los contornos de temperatura y vectores de velocidad del acero, así como la trayectoria que experimenta el acero líquido en el molde. A continuación, se destacan los siguientes aspectos:

- Producto del gradiente de temperatura, la fundición comenzó a crear una costra solidificada cerca del área del menisco, la cual aumentó el espesor en la dirección de colada hasta la salida del molde.

- El valor máximo del espesor de la costra a la salida del molde estuvo en 0,016 m, siendo un valor suficiente como para evitar el desgarro del acero líquido en el proceso.

- A la salida de SEN el acero líquido fluye con una velocidad de $0,8 \mathrm{~m} / \mathrm{s}$, alcanzando gran profundidad en el centro de la palanquilla, lo que crea un efecto de reflujo no favorable para la eliminación de posibles inclusiones y la disipación del sobrecalentamiento en el molde.

- La velocidad del flujo de acero en la zona superior del molde estuvo por debajo de los $0,2 \mathrm{~m} / \mathrm{s}$, lo que caracteriza a un flujo con bajas fluctuaciones y menor probabilidad de atrapamiento de escoria de polvos coladores, pero un alto riesgo de solidificación del menisco. 


\section{Referencias}

[1] Y. González-Rondón, J. E. Rengel-Hernández, Comportamiento termo fluidodinámico del acero en un molde de colada continua: una revisión, TecnoLógicas, vol. 24, nro. 51, e1856, (2021) [2] J. Belisario, Evaluación de la efectividad del proceso de colada en la reducción de defectos de salpicaduras en las palanquillas producidas en la acería $150 \mathrm{TM}$ de Sidor, Tesis de grado en ingeniería industrial. Universidad Nacional Experimental de Guayana, (2011).

[3] A. Cruz, O. Hernández, A. Moreno y M. Vargas, Caracterización de fundentes para molde de colada continua de acero, Acta Universitaria, vol. 17, núm. 1, enero-abril. Universidad de Guanajuato, México, (2007). pp. 52-58.

[4] V. Chang and P. Bolsaitis, Símplified model for heat transfer and solidification in continuous casting, Latin American Journal of Metallurgy and Materials, Vol. 2, N² 2, (1982).

[5] M. Najera, Análisis del flujo de fluidos y transferencia de calor sobre la calidad de palanquillas de acero, Tesis de grado de doctor en ciencias en metalurgia y materiales, Instituto Politécnico Nacional, México, (2010).

[6] C. Cicutti, Transferencia de calor en la colada continua de aceros, I parte, el molde, Revista Metalurgia, Madrid, (1977). pp. 333-344.

[7] B. Pereira, J. Castro, A. Silva, y J. Duran, Modelado del proceso de colada continua de aceros libres de intersticios, Revista Información tecnológica, Vol. 21 No. 6, (2010).

[8] J. Adilson, B. Amaral, R. Sampaio, E. Mendes and I. Leão, Numerical study of turbulent flows and heat transfer in coupled industrial-scale tundish of a continuous casting material in steel production, IntechOpen, Chapter 16, (2018).

[9] K. Mills, P. Ramirez, P. Lee, B. Santillana, B. Thomas and R. Morales, Looking into continuous casting mould. Ironmaking and Steelmaking, Vol. 41 No. 42, (2014).

[10] M. Tkadlečková, L. Válek, L. Socha, M. Saternus, J. Pieprzyca, T. Merder, K. Michalek and M. kováč M. Study of solidification of continuously cast steel round billets using numerical modelling. Arch. Metall. Mater., Vol. 61, No 1. (2016). pp. 221-226.

[11] D. Jiang, M. Zhu and L. Zhang, Numerical Simulation of Solidification Behavior and Solute Transport in Slab Continuous Casting with S-EMS, Metals, 9, 452, (2019).

[12] M. Long, H. Chen, D. Chen, S. Yu, B. Liang y H. Duan, A Combined Hybrid 3-D/2-D Model for Flow and Solidification Prediction during Slab Continuous Casting, Metals, 8, 182, (2018).

[13] X. Zhang, W. Chen, and L. Zhang, A coupled model on fluid flow, heat transfer and solidification in continuous casting mold, China Foundry. Vol. 14, No. 5, (2017).

[14] F. Saldaña-Salas, E. Torres-Alonso, J. Ramos-Banderas, G. Solorio-Díaz and C. HernándezBocanegra, Analysis of the Depth of Immersion of the Submerged Entry Nozzle on the Oscillations of the Meniscus in a Continuous Casting Mold, Metals 9, 596, (2019).

[15] P. Ni, M. Ersson, L. Ingemar Jonsson, T. Zhang and P. Jönsson, Numerical Study on the Influence of a Swirling Flow Tundish on Multiphase Flow and Heat Transfer in Mold, Metals 8, $368,(2018)$.

[16] W. Zhang, S. Luo, Y. Chen, W. Wang and M. Zhu, Numerical Simulation of Fluid Flow, Heat Transfer, Species Transfer, and Solidification in Billet Continuous Casting Mold with M-EMS. Metals. 9, 66. (2019).

[17] L.B. Trindade, J.E.A. Nadalon, A.C. Contini, and R.H. Barroso, Modeling of solidification in continuous casting round billet with mold electromagnetic stirring (M-EMS). Steel Res. Int. (2017), 88, 1600319.

[18] X. Sun, B. Li, H. Lu, Y. Zhong, Z. Ren and Z. Lei, Steel/Slag Interface Behavior under Multifunction Electromagnetic Driving in a Continuous Casting Slab Mold, Metals 9, 983, (2019).

[19] X. Yan, B. Jia, Q. Wang, S. He, and Q. Wang, Mold nonsinusoidal oscillation mode and its effect on slag infiltration for lubrication and initial shell growth during steel continuous casting, Metals 9, 418, (2019). 
[20] T.H. Shih, W.W. Liou, A. Shabbir, Z. Yang, and J. Zhu, Anew k-e eddy-viscosity model for high Reynolds number turbulent flows-Model development and validation, Computers Fluids, 24:227-38. (1995).

[21] M.R.R.I. Shamsi and S.K. Ajmani, Three dimensional turbulent fluid flow and heat transfer mathematical model for the analysis of a continuous slab caster. ISIJ International, 47:433-42. (2007).

[22] M. Bielnicki, J. Jowsa, and A. Cwudziński, Multiphase numerical model of molten steel and slag behavior in the continuous casting mould. Archives of Metallurgy and Materials, 60:257-62. (2015).

[23] P.K. Jha, S. K. Dash, and S. Kumar, Fluid flow and mixing in a six strand billet caster tundish: A parametric study, ISIJ International, 41:1437-46. (2001).

[24] P.K. Jha, and S.K. Dash, Employment of different turbulence models to the design of optimum steel flows in a tundish, International Journal of Numerical Methods for Heat \& Fluid Flow, 14:953-79. (2004).

[25] B. Thomas, Continuous Casting of Steel, Chap.15, ed. by O. Yu, Marcel Dekker, New York, (2000).

[26] B. Thomas and L. Zhang, Mathematical Modeling of Fluid Flow in Continuous Casting, ISIJ International, Vol. 41, No. 10, (2001), pp. 1181-1193. 